\title{
Revisão bibliográfica e análises de visualizações sobre Kingsleya attenboroughi \\ Pinheiro \& Santana, 2016 (Crustacea: Decapoda: Brachyura: Pseudothelphusidae)
}

Bibliographical review and analysis of visualizations about Kingsleya attenboroughi Pinheiro \&

\author{
Santana, 2016 (Crustacea: Decapoda: Brachyura: Pseudothelphusidae)
}

Revisión bibliográfica y análisis de visualizaciones sobre Kingsleya attenboroughi Pinheiro \&

Santana, 2016 (Crustacea: Decapoda: Brachyura: Pseudothelphusidae)

Recebido: 20/04/2021 | Revisado: 27/05/2021 | Aceito: 11/05/2021 | Publicado: 27/05/2021

Raimundo Samuel Leite Sampaio ORCID: https://orcid.org/0000-0002-1902-3064 Universidade Regional do Cariri, Brasil E-mail: samsampaio@ @otmail.com Junior Fernandes de Lima ORCID: https://orcid.org/0000-0003-2809-6537 Universidade Regional do Cariri, Brasil E-mail: jrlimafernandes@hotmail.com

Francisco Ilosmar Fernandes Júnior ORCID: https://orcid.org/0000-0003-1989-6479

Centro Universitário de Juazeiro do Norte, Brasil

E-mail: juniorfernandesgatopreto@hotmail.com Maraiza Gregorio de Oliveira

ORCID: https://orcid.org/0000-0002-2855-641X Universidade Regional do Cariri, Brasil E-mail: maraaiza0104@hotmail.com Dennis Bezerra Correia ORCID: https://orcid.org/0000-0002-7782-4767 Universidade Regional do Cariri, Brasil E-mail: denniscorreia40@gmail.com

Pedro Hudson Rodrigues Teixeira

ORCID: https://orcid.org/0000-0001-5909-7642 Universidade Regional do Cariri, Brasil E-mail: pedrohudson@yahoo.com.br João Paulo Camilo de Oliveira

ORCID: https://orcid.org/0000-0003-0286-1149 Universidade Regional do Cariri, Brasil

E-mail: camilodeoliveirajoaopaulo35@gmail.com Maria Haiele Nogueira da Costa

ORCID: https://orcid.org/0000--0002-0316-0830 Universidade Regional do Cariri, Brasil E-mail: haielecosta@gmail.com

Maria Ivaneide Rocha

ORCID: https://orcid.org/0000-0002-0312-9315 Universidade Regional do Cariri, Brasil E-mail: ivaneidemaria@bol.com.br

José Iago Muniz

ORCID: https://orcid.org/0000-0003-4062-3520

Universidade Regional do Cariri, Brasil E-mail: joseiagomuniz@gmail.com

Ginna Gonçalves Pereira

ORCID: https://orcid.org/0000-0002-0267-2615

Universidade Regional do Cariri, Brasil

E-mail: ginna.goncalves27@gmail.com

Nayane Kely Silva Sampaio

ORCID: https://orcid.org/0000-0001-6310-5529

Universidade Regional do Cariri, Brasil

E-mail: nayane-kelly@ hotmail.com

\section{Resumo}

Kingsleya attenboroughi, conhecido popularmente como "Guajá", é uma espécie de caranguejo de água doce, endêmica da Chapada do Araripe- Ceará, Brasil (predominância situada no distrito de Arajara- Município de Barbalha), região caracterizada com inúmeras fontes de água doce, e assim favorecendo condições ideais para a sobrevivência de K. attenboroughi na 
localidade. Esta pesquisa consiste em um levantamento de dados sobre a espécie, e diferentes bases científicas foram analisadas: Google acadêmico®, ScienceDirect®, Web of sciences®, e Pubmed ${ }^{\circledR}$ para obter as informações. K. attenboroughi difere principalmente de suas congêneres por apresentar características próprias, como uma placa apical curta no primeiro gonópode. Poucos trabalhos foram relatados até o presente momento. Sua ecologia preocupa estudiosos uma vez que esta sobre constante ameaça de extinção. Desta forma, incentivos e pesquisas vem crescendo nesta região para evitar o colapso da biodiversidade.

Palavras-chave: Chapada do Araripe; Caranguejos de água doce; Extinção; Guajá.

\begin{abstract}
Kingsleya attenboroughi, popularly known as "Guajá", is a species of freshwater crab, endemic to Chapada do Araripe- Ceará, Brazil (predominantly located in the district of Arajara- Municipality of Barbalha), a region characterized by numerous sources of fresh water, and thus favoring ideal conditions for the survival of $K$. attenboroughi in the locality. This research consists of a survey of data about the species, and different scientific bases were analyzed: Google academic ${ }^{\circledR}$, ScienceDirect ${ }$, Web of sciences $\AA$, and Pubmed ${ }^{\circledR}$ to obtain the information. $K$. attenboroughi differs mainly from its counterparts in that it has its own characteristics, such as a short apical plate in the first gonopod. Few studies have been reported to date. Its ecology worries scholars as it is under constant threat of extinction. In this way, incentives and research have been growing in this region to avoid the collapse of biodiversity.
\end{abstract}

Keywords: Chapada do Araripe; Freshwater crabs; Extinction; Guajá.

\title{
Resumen
}

Kingsleya attenboroughi, conocida popularmente como "Guajá", es una especie de cangrejo de agua dulce, endémica de Chapada do Araripe- Ceará, Brasil (ubicada predominantemente en el distrito de Arajara- Municipio de Barbalha), una región caracterizada por numerosas fuentes de agua dulce, favoreciendo así las condiciones ideales para la supervivencia de $K$. attenboroughi en la localidad. Esta investigación consiste en un relevamiento de datos sobre la especie, y se analizaron diferentes bases científicas: Google academic $®$, ScienceDirect@, Web of sciences® y Pubmed@ para obtener la información. $K$. attenboroughi se diferencia principalmente de sus contrapartes en que tiene sus propias características, como una placa apical corta en el primer gonópodo. Se han informado pocos estudios hasta la fecha. Su ecología preocupa a los estudiosos, ya que está bajo constante amenaza de extinción. De esta forma, los incentivos y la investigación han ido creciendo en esta región para evitar el colapso de la biodiversidad.

Palabras clave: Chapada do Araripe; Cangrejos de agua Dulce; Extinción; Guajá.

\section{Introdução}

Kingsleya attenboroughi Pinheiro \& Santana (2016), é uma nova espécie de caranguejos de água doce, descrito recentemente, e que está sobre ameaça de extinção (Pinheiro \& Santana 2016). Esta espécie foi descrita pela primeira vez na Chapada do Araripe, sul do estado do Ceará, Brasil. Essa biodiversidade é estritamente dependente das florestas úmidas, com uma formação geográfica que percorre três estados brasileiros (Ceará, Pernambuco e Piauí), com uma extensão de 178 km e 1000 metros de altitude (Figura 1).

Além de uma Floresta Nacional (1946), apresenta inúmeras fontes de água doce, que apesar do topo da chapada contar com uma rede de drenagem superficial praticamente nula, as camadas sotopostas apresentam uma leve inclinação para leste, favorecendo a ocorrência de cursos d'água nas depressões periféricas, e classificando o estado do Ceará como o mais rico em fontes de água, favorecendo assim a presença deste animal na região do Cariri cearense. Esta Chapada é atualmente classificada como uma área de proteção ambiental (1997) e um geoparque (2006) (Brasil, 1997, 2000, 2007; Silveira, Silva, Cabral, \& Schiavetti, 2012; ICMBIO, 2021). 
Figura 1. Foto da Chapada do Araripe, sul do estado do Ceará, Brasil.

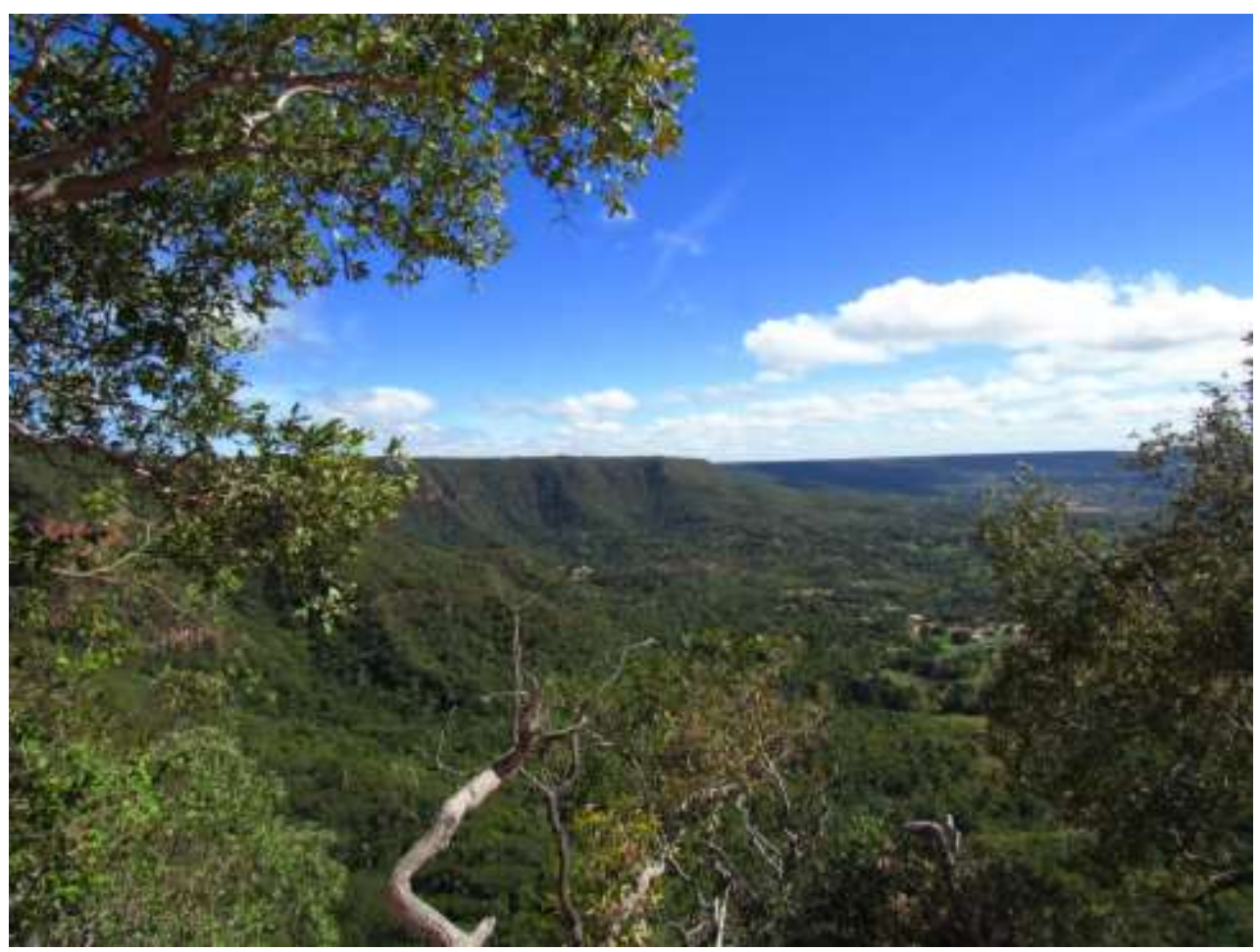

Fonte: Weverton Kariri, 2020.

Sabe-se que a disponibilidade hídrica está diretamente relacionada com a qualidade ambiental das suas áreas, e consequentemente com a sua biodiversidade local. Entretanto, trabalhos afirmam que a vegetação presente possui condições boas, porém apresentam sinais de ações antrópicas relacionadas ao uso de terras, gerando desmatamento e pontos de queimadas, além do uso exacerbado e indevido dos recursos hídricos, e esses fatores são responsáveis pela redução elevada da vazão disponível da fonte de água doce na região (SOL, 2017). Motivos estes que preocupa ecólogos e ambientalistas sobre constante ameaça na perda de espécies biológicas.

Segundo Pinheiro e Santana (2016), K. attenboroughi já está sobre um risco significativo de extinção, tomando como base os critérios B2b da IUCN, que classifica a espécie como ameaçada de extinção (EN) (IUCN, 2012). De acordo com Correia et al. (2020), até a presente data, não há ações específicas voltadas para a conservação e proteção deste caranguejo, mas que por estar localizada dentro de uma área de conservação, dá subsídios para que sua conservação seja realizada. Pesquisas sobre a espécie vêm sendo publicadas para contribuir na preservação das espécies abordando e profundando dados sobre sua ecologia e pesquisas já publicadas com este caranguejo, assim, o objetivo deste trabalho teve como base realizar um levantamento bibliográfico sobre Kingsleya attenboroughi, e trazer os mais relevantes dados cientométricos sobre esta espécie de crustáceo.

\section{Metodologia}

Levantamento de dados bibliográficas e contagem fracionária. Diferentes bases científicas foram analisadas: Google acadêmico ${ }^{\circledR}$, ScienceDirect ${ }^{\circledR}$, Web of sciences ${ }^{\circledR}$, e Pubmed ${ }^{\circledR}$ para obter as informações. Após a obtenção dos artigos nas plataformas mencionadas, com critérios de inclusão de artigos originais, dos últimos anos, escritos em inglês e português.

Um levantamento cientométrico foi realizado pra avaliar a produtividade bibliométrica, utilizando bancos de dados de acesso a documentos da ciência, "Web of Science", a escolha desta base de dados se deu por apresentar uma alta cobertura multidisciplinar, disponibilizando uma diferente ferramenta de análises cientométricas (Bar-Ilan, 2010). Os dados foram 
agrupados nas categorias: números de trabalhos publicados no decorrer dos anos e países. Utilizou-se o software GraphPad Prism versão 6 Para a produção das figuras.

Para análise fracionaria de densidade dos autores, utilizou-se uma análise gráfica dos dados bibliográficos utilizando o software VOSviewer (www.vosviewer.-com /), disponível para construir e visualizar redes cientométricas. Este programa criado por Van Eck and Waltman em 2010, é uma técnica usada para gerar co-ocorrência de termos de título de trabalhos publicados em relação ao tema (Kamdem et al., 2019). Visto que a co-ocorrências de termos indicam que é o número de vezes que dois termos ocorrem juntos em um conjunto de documentos. Todas as informações necessárias contendo informações de citações como autorias, palavras-chave, resumos e títulos foram selecionados e exportados em arquivo CSV.

\section{Resultados e Discussão}

\subsection{Análise dos dados}

Taxonomia. Este caranguejo esta inserido no filo Arthopoda, subfilo Crustacea, ordem Decapoda e na infraordem Brachyura de crustáceos que representa os "caranguejos verdadeiros", ou braquiúros, sendo composta por caranguejos e siris, da família Pseudothelphusidae. Atualmente só existem as famílias Pseudothelphusidae e Trichodactylidae, de caranguejos de água doce no Brasil (Magalhaes, 2003). Vários estudos destacam as descobertas recentes em desvendar a diversidade da fauna de caranguejos de água doce da família Pseudothelphusidae (Magalhães, 2005; Rodríguez \& Magalhães 2005).

De todas as 11 espécies já descritas conhecidas de Kingsleya, 10 ocorrem em território brasileiro (Alves \& Abrunhosa, 2010; Magalhaes, 2003). Uma espécie bem conhecida, e que é usada como chave para identificação de $K$. attenboroughi, é a K. gustavoi Magalhães, (2005), sua espécie aliada mais próxima. Um estudo comparativo entre espécies sempre aborda a $K$. gustavoi, que se diferencia por um conjunto de pequenas diferenças observadas nos parátipos (Alves \& Abrunhosa, 2010; Correia et al., 2020). Segundo Pinheiro e Santana (2016), a nova espécie difere principalmente de suas congêneres por apresentar características próprias, como uma placa apical curta no primeiro gonópode.

Habitat. Esta espécie é nativa do Norte e Nordeste da América do Sul (Pedraza e Tavares, 2015; Pinheiro e Santana, 2016), endêmica da região onde foi encontrada pela primeira vez, apresenta vários nomes populares como, "Caranguejo de água doce", "Caranguejo de rio" e "Guajá" (Correia et al., 2020). Apresenta um tamanho entre 5-7 cm (Figura 2) de sua carapaça na fase adulta do animal, e suportam uma temperatura entre $25-34{ }^{\circ} \mathrm{C}$. Seu habitat principal na região esta situada no distrito de Arajara, município de Barbalha, Ceará, Brasil (Pinheiro e Santana, 2016). Região rica em fontes naturais de água doce, fator primordial para a presença deste caranguejo na região. 
Figura 2. Tamanho do indivíduo adulto de Kingsleya attenboroughi.

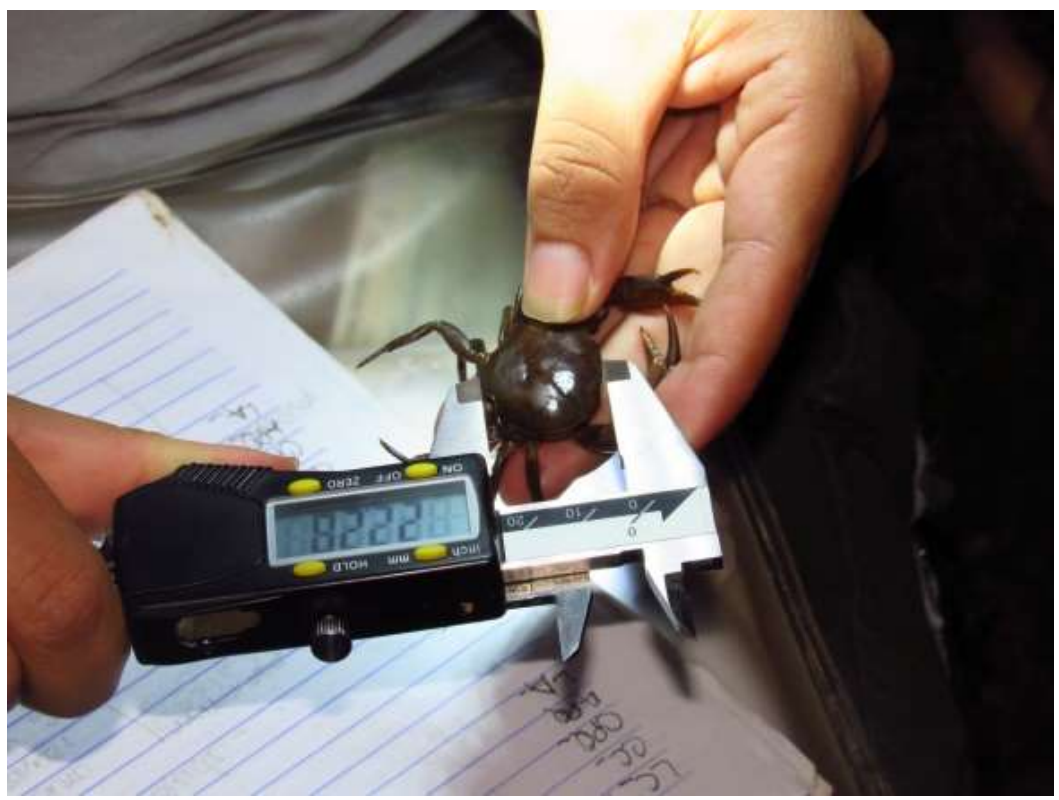

Fonte: Weverton Kariri, 2020.

Características descritivas. Como demonstrado nas figuras 3A-D, esta espécie apresenta uma carapaça elipsoide, angulada, e entre os lóbulos pós-frontais, fracamente côncavos, dorsalmente lisa e fossetas gástricas distintas na região metagástrica. Dente orbital interno bem desenvolvido, amplamente triangular, ocluindo parcialmente a cavidade orbital. Margem anterolateral da carapaça com depressão bem distinta logo atrás do ângulo exorbital, seguida por um conjunto de dentes diminutos aumentando de tamanho da porção anterior para posterior; margem póstero-lateral lisa, pouco definida (Pinheiro e Santana, 2016).

Figura 3. Caranguejo Kingsleya attenboroughi.
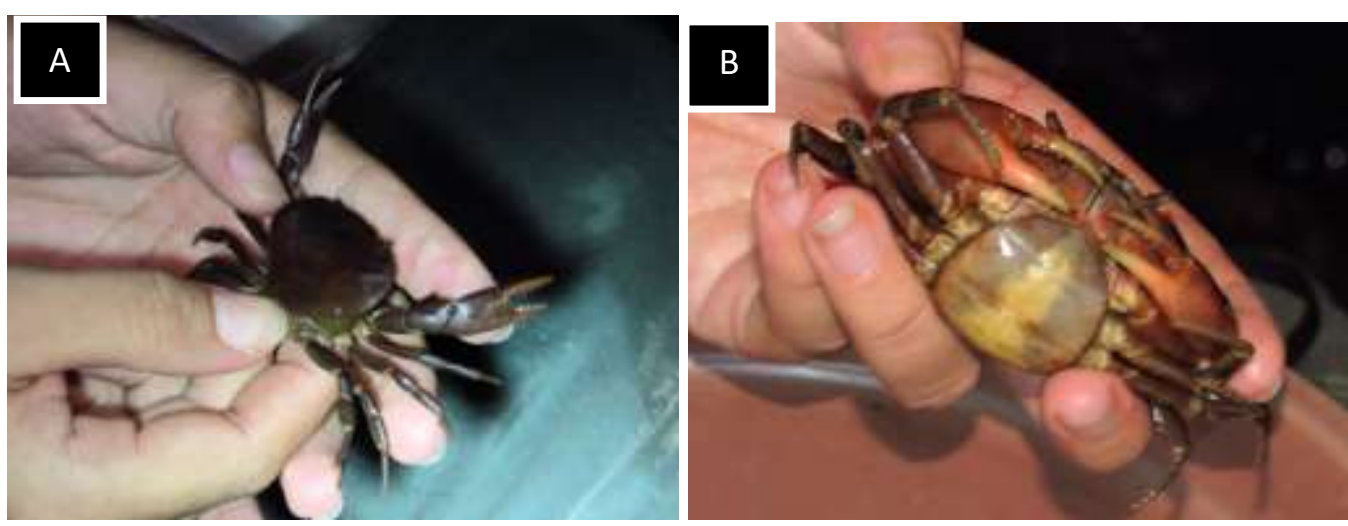


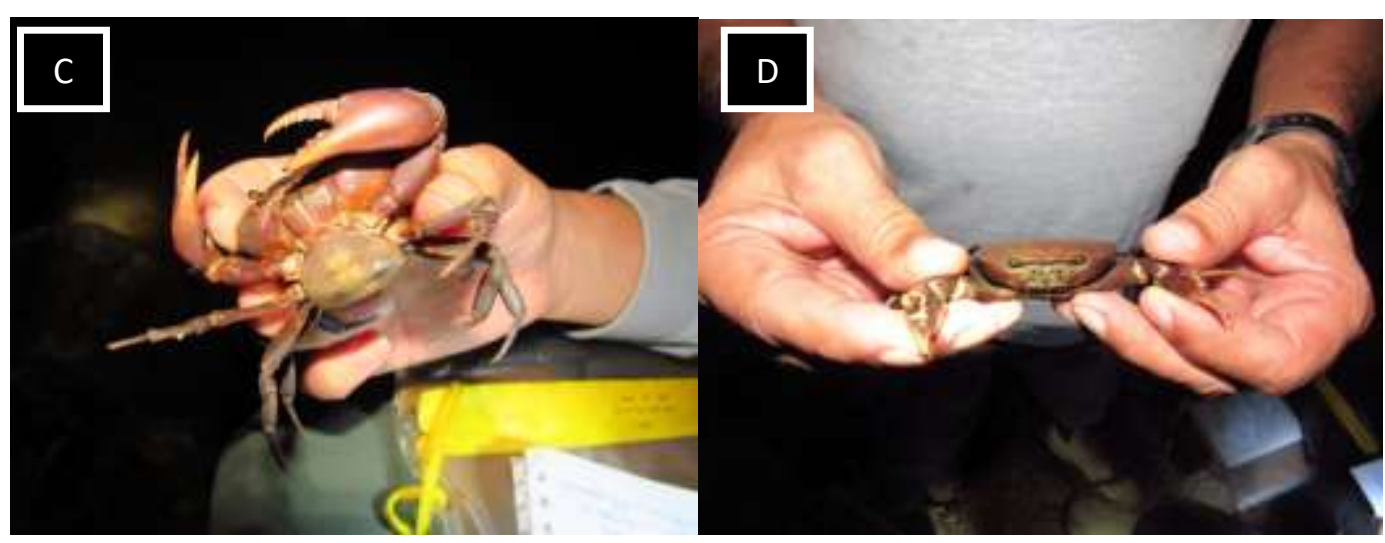

Legenda: A. Vista dorsal; B. Vista ventral com garras recuadas; C. Vista ventral com as garras abertas; D. Vista frontal. Fonte: Weverton Kariri, 2020.

\subsection{Análises de visualizações}

Anos e tipos de trabalhos. Entre os trabalhos analisados, 92,1\% são artigos de revisão e 7,9\% trabalhos diversos como trabalhos de conferências, capítulo de livro, erratas e editoria. Entre os artigos, $86 \%$ dos documentos foram citados pelo menos uma vez e $14 \%$ dos itens não apresentavam qualquer citação. Como demonstrado na figura 4, o ano de 2020 se destaca com maior índice de dados de publicação.

Figura 4. Anos de publicações com Kingsleya attenboroughi, dados fornecidos pela base Web of Science.

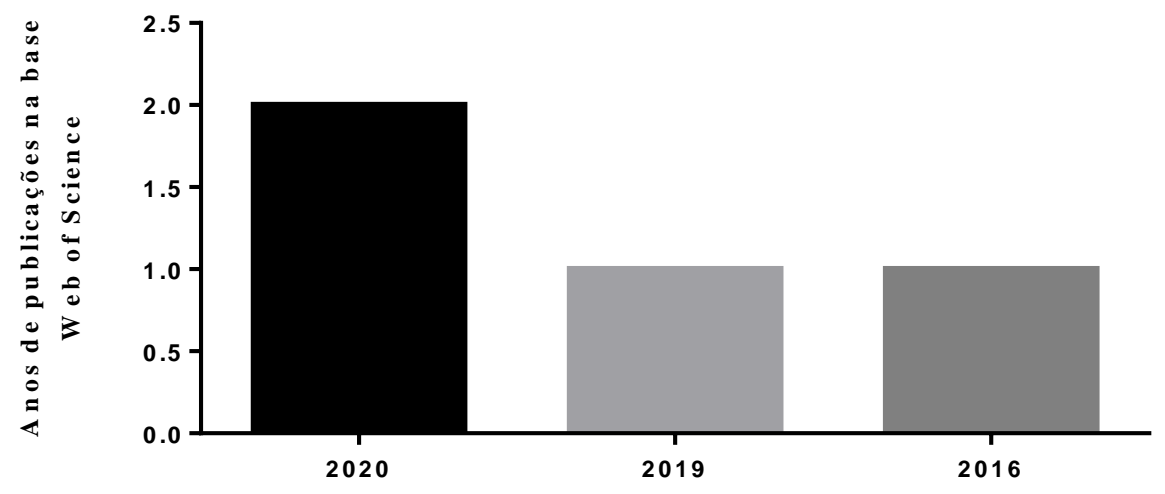

Fonte: Autores (2021).

Análise dos cluster. A Figura 5 demonstra a rede de maior densidade de autorias dos trabalhos publicados de maior citação, esses dados foram mapeados pela rede de autores usando o software VOSviewer, o número mínimo de documentos publicados por um autor foi considerado 3 documentos. O maior conjunto de itens conectados consiste nos trabalhos de Magalhaes C., Pinheiro A.P. e Tavares M., Pedraza M. ambos citados ao longo desta revisão. 
Figura 5. Rede de densidade das autorias.

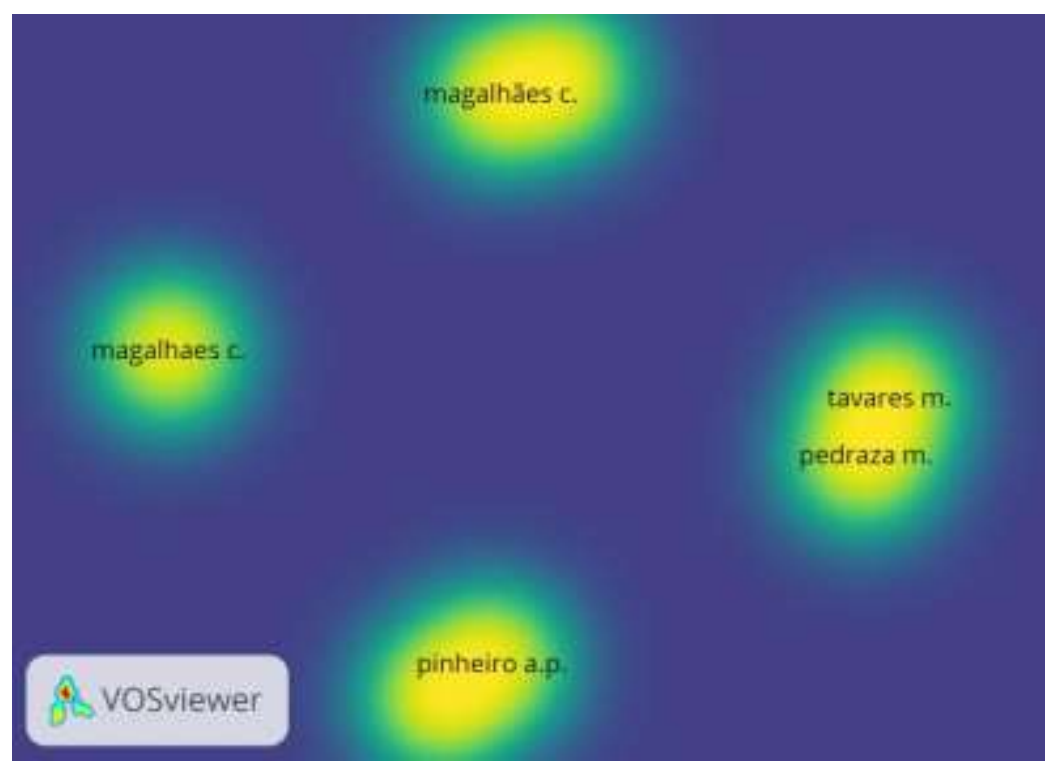

Legenda: As cores dos autores da rede indicam agrupamentos identificados pelo VOSviewer.

Fonte: Autores (2021).

Os dados de densidade abordam a importância dos trabalhos e colaboração entre pesquisadores, ilustrando a importância do nó na rede de coautoria de autores. Sabendo-se que as coautorias são as formas mais tangíveis de colaboração em pesquisa (Giuliani, Petris, \& Nico, 2010). A parceria e diversidade mundial de autores que escrevem em uma determinada área e assunto são importantes para formar o sistema global de produção de conhecimento, destacando a importância às redes de coautoria (Giuliani, Petris, \& Nico, 2010; Zare-Farashbandi, Gerae, \& Siamaki, 2014). Assim a produção científica, em termos gerais de números, tanto de trabalhos quanto os aspectos de citações são afetados positivamente por filiações, centros, universidades e outros.

Filiações. O Brasil lidera o ranking de publicações, com 99,31\% de publicações. Esse destaque do Brasil pode ser justificado pelo caranguejo ser endêmico da região do Cariri (Ceará- Brasil), fator que contribui para os estudos ecológicos e obtenção do animal, uma vez que pode ser encontrada nos córregos de água doce nas proximidades.

\section{Conclusão}

Estudos desta natureza fortalecem a pesquisa sobre o caranguejo Kingsleya attenboroughi, contribuindo para explanação da sua ecologia e sua importância para biodiversidade local. Realçando a importância da preservação da flona, flora e recursos hídricos da Chapada do Araripe. Mais estudos devem ser realizados para contribuir significativamente para a preservação desta e de outras espécies desta Floresta.

\section{Referências}

Alves, S. T. M., Abrunhosa, F. A., \& Lima, J. D. F. (2010). Foregut morphology of Pseudothelphusidae and Trichodactylidae (Decapoda: Brachyura) from northeastern Pará, Brazil. Zoologia, 27(2), 228-244.

Bar-Ilan, J. (2010). Citations to the "Introduction to informetrics" indexed by WOS, Scopus and Google Scholar. Scientometrics. 82(3), $495-506$.

Brasil. (1997). Decreto de 04 de agosto de 1997. Dispõe sobre a criação da Área de Proteção Ambiental da chapada do Araripe, nos Estados do Ceará, Pernambuco e Piauí e dá outras providências. Diário Oficial. Brasília. 
Brasil. (2000). Lei $n^{\circ} 9.985$ de 18 de julho de 2000. Regulamenta o art. 225, $1^{\circ}$, incisos I, II, III e VIII d Constituição Federal, institui o Sistema Nacional de Unidades de Conservação e dá outras providências. Acesso em 12, Fev., 2021, http://www2.camara.leg.br/legin/ fed/lei/2000/lei-9985-18-julho-2000-359708norma-pl.html

Brasil. (2007). Lei nº 11.516, de 28 de agosto de 2007. Criação do Instituto Chico Mendes de Conservação da Biodiversidade. Diário Oficial. Brasília

Correia, D. B., Freita, F. R. V., Oliveira, A. H., Correia, D. B., Teixeira, P. H. R., Soares, T. R. C... \& Pinheiro, A. P. (2020). Ethnobiology of Kingsleya attenboroughi Pinheiro \& Santana crab 2016 in the Environmental Protection Area of Chapada do Araripe. Research, Society and Development, 9(12), p. e19091210827.

Giuliani, F., Petris, M. P., \& Nico, G. (2010). Assessing scientific collaboration through coauthorship and content sharing. Scientometrics, 85(1), 13-28.

ICMBIO. (2021). Instituto Chico Mendes de Conservação da Biodiversidade. https://www.icmbio.gov.br/portal/unidadesdeconservacao/biomasbrasileiros/caatinga/unidades-de-conservacao-caatinga/2110-apa-da-chapada-do-araripe.

IUCN. (2012). International Union for Conservation of Nature. Guidelines for Application of IUCN Red List Criteria at Regional and National Levels: Version 4.0. Gland, Switzerland and Cambridge, UK: IUCN

Kamdem, J. P., Duarte, A. E., Lima, K. R. R., Rocha, J. B. T., Hassan, W., Barros, L. M., \& Tsopmo, A. (2019). Research trends in food chemistry: A bibliometric review of its 40 years anniversary (1976-2016). Food Chemistry, 294, 448-457.

Kamdem, J. P., Abolaji, A. O., Roos, D. H., Calabró, L., Barbosa, N. V., Souza, D. O., \& Rocha, J. B. T. (2016). Scientifc Performance of Brazilian Researchers in Pharmacology with grants from CNPq: A comparative study within the Brazilian categories. Annals of the Brazilian Academy of Sciences, 28, $1735-1742$.

Magalhães C. (1986). Revisão taxonômica dos caranguejos de água doce brasileiros da família Pseudothelphusidae (Crustacea, Decapoda). Amazoniana, 9(4), 609-636.

Magalhães C. (1990). A new species of the genus Kingsleya from Amazonia, with a modified key for the Brazilian Pseudothel phusidae (Crustacea: Decapoda: Brachyura). Zoologische Mededelingen, 63(21), 275-281.

Magalhães C. (2003). The occurrence of freshwater crabs (Crustacea: Decapoda: Pseudothelphusidae, Trichodactylidae) in the Rio Xingu, Amazon Region, Brazil, with description of a new species of Pseudothelphusidae. Amazoniana, 17(3/4), 377-386.

Magalhães, C. (2005) A new species of freshwater crab (Decapoda: Pseudothelphusidae) from the southeastern Amazon Basin. Nauplius, 12 , 99-107.

Pedraza, M. \& Tavares, M. (2015) A new species of freshwater crab of the genus Kingsleya attenboroughi, 1897 (Crustacea: Brachyura: Pseudothelphusidae) from Amazonia, Brazil. Zootaxa, 4032(4),444-450.

Pinheiro, A. P., \& Santana, W. (2016). A new and endangered species of Kingsleya attenboroughi, 1897 (Crustacea: Decapoda: Brachyura: Pseudothelphusidae) from Ceará, northeastern Brazil. Zootaxa, 4171(2), 365-373.

Rodríguez, G. \& Magalhães, C. (2005) Recent Advances in the biology of the Neotropical freshwater crabs family Pseudothelphusidae (Crustacea, Decapoda, Brachyura). Revista Brasileira de Zoologia, 22, 354-365.

Silveira, A. C., Silva, A. C., Cabral, N. R. A. J., \& Schiavetti, A. (2012). Análise de efetividade de manejo do Geopark Araripe - Estado do Ceará, São Paulo, UNESP, Geociências, 31(1), 117-128.

SOL. (2017). Sistema de Outorga e Licença. Secretaria de Recursos Hídricos. Companhia de Gestão de Recursos Hídricos. Fortaleza - CE.

Van Eck, N. J., \& Waltman, L. (2010). Software survey: VOSviewer, a computer program for bibliometric mapping. Scientometrics, 84(2), 523-538.

Zare-Farashbandi, F., Geraei. E., Siamaki, S. (2014). Study of coauthorship network of papers in the Journal of Research in Medical Sciences using social network analysis. Journal of Research in Medical Sciences, 19(1), 41-46. 